\title{
Coccygectomy provides partial relief for tailbone pain
}

\author{
Patrick M. Foye
}

Received: 14 June 2010 / Accepted: 24 June 2010 / Published online: 13 July 2010

(C) Springer-Verlag 2010

As a physician who treats hundreds of coccydynia patients per year, I praise your journal and authors Gáspár et al. [1] on their excellent research study titled, "Coccygectomy has a favourable effect on the intensity, manifestation, and characteristics of pain caused by coccygodynia: a retrospective evaluation of 34 patients followed for 3-18 years".

Their research documents very comprehensive and wonderfully long-term (3-18 years) follow-up after coccygectomy. Of their 34 coccygectomy patients, excellent relief was obtained in $12(35 \%)$, good relief in $16(47 \%)$, no change in $5(15 \%)$, and worsening in 1 patient (3\%). Overall, these are admirable results for a condition that is often so resistant to treatment.

The data in this study show a crucial point that patients and doctors should realize. Specifically, while most patients eventually experience substantial improvement compared with preoperative pain scores, unfortunately only $20 \%$ of patients (7 out of 34) obtain "complete" relief of their tailbone pain. This is extremely important, so that patients and doctors can have realistic expectations before coccygectomies are performed. Many patients simplistically believe that once their coccyx is gone then their pain will automatically be completely gone as well, but this usually does not occur (neither in the study, nor in my clinical experience). Before coccygectomy surgery, patients should be educated about realistic expectations. Such education would likely decrease the disappointment of not having "complete" relief, and instead would most likely improve patients' postoperative satisfaction with the amount of relief that they usually do obtain.

Conflict of interest statement None.

\section{Reference}

1. Gáspár L, Jónás Z, Kiss L, Vereb G, Csernátony Z (2009) Coccygectomy has a favourable effect on the intensity, manifestation, and characteristics of pain caused by coccygodynia: a retrospective evaluation of 34 patients followed for 3-18 years. Eur J Orthop Surg Traumatol 19(6):403-407
P. M. Foye $(\bowtie)$

Coccyx Pain Service, University Hospital,

University of Medicine and Dentistry of New Jersey (UMDNJ),

New Jersey Medical School, 90 Bergen St, D.O.C. Suite 3100,

Newark, NJ 07103-2425, USA

e-mail: Patrick.Foye@UMDNJ.edu 